\title{
Hábitos alimenticios de Lagidium viscacia y Abrocoma cinerea: roedores sintópicos en ambientes altoandinos del norte de Chile
}

\author{
Food-habits of Lagidium viscacia and Abrocoma cinerea: syntopic rodents in high \\ Andean environments of northern Chile
}

ARTURO CORTÉS ${ }^{1,2}$, JAIME R. RAU ${ }^{3}$, EDUARDO MIRANDA ${ }^{1} \&$ JAIME E. JIMÉNEZ $^{3}$

\begin{abstract}
${ }^{1}$ Departamento de Biología, Facultad de Ciencias, Universidad de La Serena, Casilla 599, La Serena, Chile; e-mail2: acortes@userena.cl

${ }^{3}$ Laboratorio de Ecología, Departamento de Ciencias Básicas, Universidad de Los Lagos, Casilla 933, Osorno, Chile
\end{abstract}

\begin{abstract}
RESUMEN
Lagidium viscacia y Abrocoma cinerea son dos especies de roedores herbívoros de distribución geográfica simpátrida en los ambientes altoandinos del Norte Grande de Chile, donde la disponibilidad de alimento es escasa, fluctuante y poco predecible. Dada su sintopía, y por habitar en ambientes áridos altoandinos (Segunda Región de Chile) con escasez de recursos alimenticios, se espera que ambas especies de roedores seleccionen diferencialmente sus recursos, o segregarse en otros ejes de nicho (e.g., nicho espacial) a fin de evitar la presumible competencia explotativa por los recursos alimenticios. En este estudio evaluamos los hábitos alimenticios de estas dos especies durante dos estaciones contrastantes (invierno, 1996 y verano, 1997). Los resultados indicaron que: (1) L. viscacia consumió 10 ítemes alimenticios en invierno y siete en verano de las 12 únicas especies de plantas presentes en su ambiente. El ítem alimenticio mayormente consumido fue Stipa bomanii, con un 20,9\% (invierno 1996) y un 30,1\% (verano 1997). Otros ítemes alimenticios importantes, pero sólo consumidos en invierno, fueron Nicotiana longibracteata $(12,1 \%)$ y Parastrephia quadrangularis $(9,4 \%)$; mientras que en verano consumió: Festuca ortophylla (19,9\%). En cambio, A. cinerea consumió en invierno Baccharis tola $(73,2 \%)$, Lupinus oreophilus $(11,9 \%)$, Adesmia horrida $(3,6 \%)$ y $P$. quadrangularis $(0,4 \%)$, mientras que en verano consumió B. tola $(33,7 \%)$, A. horrida $(22,5 \%)$, L. oreophilus $(24,0$ $\%), P$. quadrangularis $(2,3 \%)$ y Ephedra rupestris $(2,9 \%)$. (2) Lagidium viscacia presentó mayor amplitud de nicho trófico que $A$. cinerea (invierno: $\mathrm{H}^{\prime}=0,67$ versus 0,25 ; verano: $\mathrm{H}^{\prime}=0,53$ versus 0,56 ). (3) En invierno, L. viscacia mostró alta selección por $N$. longibracteata $\left(\mathrm{E}_{\mathrm{i}}=0,96\right)$ y Calceolaria stellarifolia $\left(\mathrm{E}_{\mathrm{i}}=0,80\right)$ y, en menor grado, por $P$. quadrangularis $\left(\mathrm{E}_{\mathrm{i}}=0,62\right)$ y $S$. bomanii $\left(\mathrm{E}_{\mathrm{i}}=0,26\right)$, mientras que en verano seleccionó a las gramíneas $F$. orthophylla $\left(\mathrm{E}_{\mathrm{i}}=0,52\right)$ y $S$. bomanii $\left(\mathrm{E}_{\mathrm{i}}=0,42\right)$ y, en menor grado, C. stellarifolia $\left(\mathrm{E}_{\mathrm{i}}=0,22\right)$. Durante invierno A. cinerea seleccionó, L. oreophilus $\left(\mathrm{E}_{\mathrm{i}}=0,91\right)$ y $B$. tola $\left(\mathrm{E}_{\mathrm{i}}=0,35\right)$; mientras que en verano los ítemes alimenticios seleccionados fueron $L$. oreophilus $\left(\mathrm{E}_{\mathrm{i}}=0,92\right)$, A. horrida $\left(\mathrm{E}_{\mathrm{i}}=0,56\right)$ y E. rupestris $\left(\mathrm{E}_{\mathrm{i}}=0,55\right)$, contrariamente, durante este período estacional $A$. cinerea mostró cierto rechazo por $P$. quadrangularis $\left(\mathrm{E}_{\mathrm{i}}=-0,40\right)$. (4) Ambas especies tuvieron durante las dos estaciones estudiadas una baja sobreposición en sus dietas (invierno: $\mathrm{C}_{\mathrm{H}}=0,18$ y en verano $\mathrm{C}_{\mathrm{H}}=0,06$ ). En síntesis, estos roedores herbívoros sintópicos exhiben una baja tendencia a seleccionar una dieta, especialmente $L$. viscacia, que tendió a ser más generalista; no obstante, ambas especies presentaron nichos tróficos diferentes, de modo que la condición de sintopía para estas especies no implicaría necesariamente competencia explotativa por sus recursos alimenticios, al menos durante las dos estaciones analizadas en este estudio.
\end{abstract}

Palabras clave: ambiente altoandino, Abrocoma cinerea, Lagidium viscacia, roedores sintópicos, selección dietaria, sobreposición dietaria.

\section{ABSTRACT}

The mountain viscacha (Lagidium viscacia) and the Andean chinchilla rat (Abrocoma cinerea) are syntopic species in the Andean highlands of northern Chile. Given that in this environment food availability is scarce, we predict that these rodents would select different food items in order to avoid presumable competition for food. We described the food habits of mountain viscachas and chinchilla rats in the highlands of northern Chile during contrasting seasons: winter (1996) and summer (1997). Results indicate that: (1) Out of the 12 plant species available, L. viscacia consumes 10 species during the winter and seven during the summer. The main species eaten was Stipa bomanii, making 20.9 and $30.1 \%$ of the winter and summer diets, respectively. Other important plants consumed only during the winter were Nicotiana longibracteata (12.1\%) and Parastrephia quadrangularis (9.4\%). In turn, Festuca ortophylla (19.9\%) was eaten only during the summer. The main plants consumed by A. cinerea during the winter were Baccharis tola (73.2 $\%$ ) and Lupinus oreophilus (11.9\%), whereas during the summer these were B.tola (33.7 \%), Adesmia horrida (22.5 $\%$ ), and L. oreophilus $(24.0 \%$ ); (2) L. viscacia had a broader trophic niche than A. cinerea (winter H' $=0.67$ versus 0.25 ; summer $\mathrm{H}^{\prime}=0.53$ versus 0.56 , respectively). (3) During winter, L. viscacia showed high selection for $N$. 
longibracteata $\left(\mathrm{E}_{\mathrm{i}}=0.96\right)$ and Calceolaria stellarifolia $\left(\mathrm{E}_{\mathrm{i}}=0.80\right)$, and lower for P. quadrangularis $\left(\mathrm{E}_{\mathrm{i}}=0.62\right)$ and $S$. bomanii $\left(\mathrm{E}_{\mathrm{i}}=0.26\right)$, whereas during summer it selected the graminoids $F$. orthophylla $\left(\mathrm{E}_{\mathrm{i}}=0.52\right)$ and $S$. bomanii $\left(\mathrm{E}_{\mathrm{i}}\right.$ $=0.42)$. In contrast, A. cinerea selected L. oreophilus $\left(\mathrm{E}_{\mathrm{i}}=0.91\right)$ and B. tola $\left(\mathrm{E}_{\mathrm{i}}=0.35\right)$ during the winter and $L$. oreophilus $\left(\mathrm{E}_{\mathrm{i}}=0.92\right)$, A. horrida $\left(\mathrm{E}_{\mathrm{i}}=0.56\right)$, and E. rupestris $\left(\mathrm{E}_{\mathrm{B}}=0.55\right)$ during the summer. During summer season $P$. quadrangularis was rejected $\left(\mathrm{E}_{\mathrm{i}}=-0.40\right)$ by $A$. cinerea. (4) Both rodents showed low food overlap during both seasons $\left(\mathrm{C}_{\mathrm{H}}=0.18\right.$ and 0.06 for winter and summer, respectively). In sum, these herbivorous species live in the same place and have rather broad diet selection, especially L. viscacia, which is more generalist than A. cinerea. However, they have distinctive trophic niches so that, unless during the sampled seasons, competition for food is unlikely.

Key words: Abrocoma cinerea, diet selection, diet overlap, highland environment, Lagidium viscacia, syntopic rodents.

\section{INTRODUCCIÓN}

La dieta de los mamíferos herbívoros es considerada como una de las más complejas, en comparación con otros consumidores (Belovsky 1978, Westoby 1978, Owen-Smith \& Novellie 1982). Este punto de vista se sustenta en que los herbívoros consumen alimentos en alta abundancia, pero de baja calidad, que a menudo carecen de los nutrientes esenciales (Westoby 1978), de modo que requieren la ingestión complementaria de diferentes ítemes alimenticios para mantener el balance nutricional (Belovsky 1978, 1984). Además, los herbívoros frecuentemente están obligados a consumir plantas que contienen compuestos tóxicos, los cuales pueden limitar el grado de selección de sus dietas (Harborne 1982, Crawley 1983).

La información de la dieta de los animales es un componente importante para la comprensión de su autoecología (Krebs 1989), dado que permite entender las relaciones tróficas entre las especies (Zimmerman 1965, Bar et al. 1984), y entre individuos y su ambiente (Kronfeld \& Dayan 1998). El estudio de la selección trófica, o preferencia alimenticia, en la cual se consideran los valores nutricionales de los ítemes alimenticios seleccionados por las especies (Petrides 1975), es de utilidad para predecir los hábitats potenciales que serían ocupados por las especies (Drickamer 1971), y la segregación espacial a escala geográfica entre especies simpátridas (Drickamer 1970) $y$, en casos de estudios más complejos, permite determinar el impacto de las poblaciones de herbívoros sobre la cubierta vegetacional (Petrides 1975).

Una extensión de lo anterior es que los estudios dietarios de animales silvestres han cobrado importancia en los planes de conservación de especies y planificación de reservas naturales (Cole et al. 1995), reintroducción de especies exterminadas a sus hábitats naturales (Lundie-Jenkins et al. 1993, Renssen \& Vogel 1993, Bright \& Morris 1994, Hartman 1994) y en el desarrollo de técnicas de control biológico (Beg et al. 1994, Jiménez et al. 1994, Tobin et al. 1994).
Numerosos métodos y técnicas han sido utilizados para determinar los ítemes alimenticios que consumen y/o seleccionan los animales herbívoros, dentro de los que se incluyen las observaciones de campo (Davison \& Graetz 1957, Holechek et al. 1982a) e identificación de los ítemes alimenticios consumidos a partir del análisis de mollejas, buches, estómagos y fecas (Dalke et al. 1942, Sparks \& Malechek 1968, Severson 1981, Holechek et al. 1982b, Alipayou et al. 1993, Lewis 1994). El análisis del material fecal para el estudio de la dieta de animales herbívoros ha sido ampliamente utilizado (Fitzgerald \& Waddington 1979, Severson 1981, Morales 1985), técnica que involucra el reconocimiento microhistológico de tejidos vegetales incluidos en las fecas (Holechek et al. 1982a). Esta técnica permite estimar el ítem trófico consumido presente en una área determinada (Hurst 1992). Esta técnica se utiliza preferentemente cuando se desea evitar el sacrificio de los animales, especialmente cuando se trata de especies en peligro de extinción o protegidas por leyes (Holecheck et al. 1982a, Kronfeld \& Dayan 1998).

Lagidium viscacia (Molina 1782) y Abrocoma cinerea (Thomas 1919) son roedores sintópicos, a escala ecológica, en ambientes áridos altoandinos (3.000 a $5.000 \mathrm{~m}$ de altitud), donde los recursos tróficos son escasos, fluctuantes y poco predecibles (Marquet et al. 1998). Lagidium viscacia presenta una distribución más amplia a lo largo de la cordillera de Los Andes, desde el sur del Perú al centro sur de Chile y de Argentina (Redford \& Eisenberg 1992), mientras que $A$. cinerea se distribuye desde el suroeste del Perú al extremo norte de Chile (Segunda Región de Chile) y por el oriente de la cordillera de Los Andes (Argentina), desde la provincia de Salta hasta el sur de Mendoza (Redford \& Eisenberg 1992). En Chile, L. viscacia y A. cinerea son especies consideradas dentro de las categorías de conservación correspondientes a "vulnerable" e "insuficientemente conocida", respectivamente (Glade 1993). Estudios de la biología de estas especies son escasos, probablemente debido al reducido número poblacional en que se encuentran en la 
actualidad, causado probablemente por la intensiva caza que fueron objetos en el pasado (Pearson 1948, Mann 1978, Campos 1996). Las especies que aquí estudiamos presentan una marcada sintopía, coexistiendo en roqueríos de los sectores altoandinos áridos del Norte Grande de Chile (Segunda Región de Chile). Descripciones sobre los hábitos alimenticios de L. viscacia y A. cinerea son extremadamente generales, indicando que son especies herbívoras (Pearson 1948, Mann 1978).

Dada su sintopía, y por habitar en ambientes áridos altoandinos (Segunda Región de Chile) con escasez de recursos alimenticios, se espera que ambas especies de roedores seleccionen diferencialmente sus recursos, o segregarse en otros ejes de nicho (e.g., nicho espacial) a fin de evitar presumible competencia explotativa por los recursos alimenticios. En este estudio evaluamos los hábitos alimenticios de estas dos especies durante dos estaciones contrastantes (invierno, 1996 y verano, 1997) en una localidad del altiplano chileno.

\section{TABLA 1}

\section{Cobertura foliar en el hábitat de Lagidium viscacia y Abrocoma cinerea, durante invierno 1996 y verano 1997 (sector altoandino de la localidad de Talabre Viejo, Segunda Región de Chile)}

Plant cover in the habitat of Lagidium viscacia and Abrocoma cinerea during winter 1996 and austral summer 1997 (puna plateau at Talabre Viejo, Segunda Región de Chile)

\begin{tabular}{lrr}
\hline Familia y & \multicolumn{2}{c}{ Cobertura foliar (\%) } \\
especie & Invierno & Verano \\
\hline Compositae & & \\
$\quad$ Artemisia copa & 3,0 & 7,8 \\
Baccharis tola & 18,2 & 22,0 \\
$\quad$ Parastrephia quadrangularis & 2,0 & 4,2 \\
Ephedraceae & & \\
$\quad$ Ephedra rupestris & 0,5 & 0,7 \\
Gramineae & & \\
$\quad$ Festuca orthophylla & 5,0 & 7,2 \\
$\quad$ Stipa bomanii & 11,2 & 14,2 \\
Papilionaceae & & \\
$\quad$ Adesmia horrida & 2,2 & 5,0 \\
$\quad$ Lupinus oreophilus & 0,3 & 0,8 \\
Scrophulariaceae & & \\
$\quad$ Calceolaria stellariifolia & 0,5 & 1,6 \\
Solanaceae & & \\
$\quad$ Nicotiana longibracteata & 0,3 & 0,5 \\
$\quad$ N. petunioides & 0,3 & 0,5 \\
Verbenaceae & & \\
$\quad$ Junellia seriphioides & 3,0 & 3,5 \\
Suelo desnudo & 53,5 & 32,0 \\
\hline
\end{tabular}

\section{MATERIALES Y MÉTODOS}

\section{Sitio de estudio}

El estudio de la dieta de los roedores Lagidium viscacia (Chinchillidae) y Abrocoma cinerea (Abrocomidae) se realizó durante invierno (agosto de 1996) y verano (marzo de 1997), a partir de fecas frescas, que se diferenciaron por tamaño y forma, previamente identificadas a partir de individuos capturados de ambas especies. Dichas fecas se colectaron en sectores altoandinos $(3.660 \mathrm{~m}$ de altitud) del Norte de Chile (Segunda Región de Chile), ubicados a $40 \mathrm{~km}$ al sureste de la localidad de Talabre Viejo (2321'22'S, 6748'55.6”O).

El sitio de estudio se caracterizó por presentar temperaturas medias similares durante invierno y verano $\left(8,5\right.$ y $8,7^{\circ} \mathrm{C}$, respectivamente). Sin embargo, la humedad media en invierno y verano fueron de 6,7 y 40,3\%, respectivamente (A. Cortés resultados no publicados). La mayor humedad en verano estaría asociada principalmente a los efectos del "invierno boliviano", fenómeno climático caracterizado porque las precipitaciones ocurren preferentemente durante el período estival (Grosjean et al. 1991). La vegetación predominante del sitio de estudio correspondió principalmente a especies arbustivas, Baccharis tola y Adesmia horrida y la herbácea perenne Stipa bomanii (gramínea).

\section{Cobertura de la vegetación}

La cobertura foliar de las especies de plantas se obtuvo de acuerdo a la técnica del punto de intersección (Mueller-Dombois \& Ellerberg 1974). La cobertura de plantas herbáceas y arbustivas durante invierno y verano se determinó usando 8 transectos de $15 \mathrm{~m}$, elegidos al azar. Cada transecto se dividió en 75 puntos, separados cada $20 \mathrm{~cm}$, con un total de muestra de 600 puntos. Para el tratamiento de los resultados los valores de cobertura sólo se sumaron, obteniéndose un único valor global por especie.

\section{Colección de referencia}

En el sitio de estudio se recolectaron hojas, tallos y flores de las diferentes especies de plantas presentes en el sitio de estudio (Tabla 1). La identificación al nivel de especie se realizó en el Herbario de la Universidad de La Serena. Las muestras colectadas fueron transportadas al laboratorio y secadas a $60^{\circ} \mathrm{C}$, de modo de preservar el material vegetal (Korschgen 1969, Hansson 1970). 
Los tejidos epidérmicos de estas plantas fueron usados como material de referencia para la identificación de las plantas consumidas por herbívoros (Dusi 1949, Williams 1962). El material microhistológico de referencia se obtuvo mediante la técnica de Sparks \& Malechek (1968), con algunas modificaciones en los tiempos de decoloración del tejido.

\section{Recolección de fecas y preparaciones histológicas}

Se recolectaron 100 muestras de fecas frescas de L. viscacia y de A. cinerea por estación (invierno y verano). Las muestras de fecas colectadas fueron transportadas al laboratorio y secadas a 60 ${ }^{\circ} \mathrm{C}$, de modo de preservar el material (Korschgen 1969, Hansson 1970). Del total de las muestras de fecas $(n=100)$, se utilizaron 40 fecas tomadas al azar. A partir de dichas muestras de fecas se confeccionaron 10 preparaciones histológicas, usando la técnica de Williams (1962), descrita por Dizeo de Strittmatter (1984).

\section{Ítemes de alimentos consumidos}

Se analizaron diez campos por preparación histológica (10 preparaciones $=100$ campos $)$, para cada estación y especie. Las observaciones se realizaron con un microscopio Leitz Laborlux 12 , con ocular equipado con una lentilla reticulada de 7 x 7 cuadrantes. Todas las observaciones se realizaron con aumento de 36x. Los campos que contenían menos del $50 \%$ del área ocupada por estructuras epidérmicas fueron excluidos (Meserve 1981). El material epidérmico fue identificado al nivel de especie. La estimación de los ítemes alimenticios consumidos (tejidos y fibras), se basó en las mediciones del área relativa ocupada por cada ítem en el campo microscópico analizado, lo que permitió cuantificar la frecuencia de los ítemes de cada preparación histológica de la muestra de fecas (Stewart 1967).

\section{Selección dietaria}

La selección para cada ítem alimenticio consumido fue estimada suponiendo que el alimento seleccionado estuvo en proporción directa a la disponibilidad de los recursos tróficos presentes en el sitio de estudio (estimado por la cobertura foliar de la vegetación). Esta selección trófica se cuantificó usando el índice de electividad de Ivlev ( $E_{i}$, Alcoze \& Zimmerman 1973, Krebs 1989):

$$
\mathrm{E}_{\mathrm{i}}=[\mathrm{r}(\mathrm{i})-\mathrm{p}(\mathrm{i})] /[\mathrm{r}(\mathrm{i})+\mathrm{p}(\mathrm{i})]
$$

donde: $\mathrm{r}(\mathrm{i})=$ proporción del área relativa $(\%)$ del ítem i consumido y $\mathrm{p}(\mathrm{i})=$ proporción del ítem de alimento i presente en la cobertura vegetación $(\%)$. Los valores de $\mathrm{E}_{\mathrm{i}}$ fluctúan entre - 1 (= rechazo, o selección negativa) y 1 (= preferencia, o selección positiva) y un valor de $\mathrm{E}_{\mathrm{i}}$ igual a cero supone un consumo de alimento aleatorio (al azar, o en proporción a su oferta ambiental).

\section{Amplitud del nicho trófico}

La amplitud de nicho trófico se calculó mediante el índice de diversidad ecológica de ShannonWeaver (H', Perkin 1982):

$$
H^{\prime}=-A p_{i} \times \log p_{i}
$$

donde: $\mathrm{p}_{\mathrm{i}}=\mathrm{n}_{\mathrm{i}} / \mathrm{N}$, es la proporción del número total de plantas de una determinada especie $\mathrm{i}, \mathrm{n}_{\mathrm{i}}=$ número de plantas por especie y $\mathrm{N}=$ número total de plantas.

\section{Similitud de la dieta}

La similitud de la dieta, se calculó mediante el índice de Horn (1966) $\left(\mathrm{C}_{\mathrm{H}}\right.$, Cox 1990):

$$
\mathrm{C}_{\mathrm{H}}=\frac{\sum\left(\mathrm{X}_{\mathrm{i}}+\mathrm{Y}_{\mathrm{i}}\right) \log \left(\mathrm{X}_{\mathrm{i}}+\mathrm{Y}_{\mathrm{i}}\right)-\sum\left(\mathrm{X}_{\mathrm{i}} \log \mathrm{X}_{\mathrm{i}}\right)-\sum\left(\mathrm{Y}_{\mathrm{i}} \log \mathrm{Y}_{\mathrm{i}}\right)}{\left(\mathrm{N}_{\mathrm{A}}+\mathrm{N}_{\mathrm{B}}\right) \log \left(\mathrm{N}_{\mathrm{A}}+\mathrm{N}_{\mathrm{B}}\right)-\left(\mathrm{N}_{\mathrm{A}} \log \mathrm{N}_{\mathrm{A}}\right)-\left(\mathrm{N}_{\mathrm{B}} \log \mathrm{N}_{\mathrm{B}}\right)}
$$

donde: $\mathrm{X}_{\mathrm{i}}=$ número de especies $\mathrm{i}$ en la comunidad $\mathrm{A}, \mathrm{Y}_{\mathrm{i}}=$ número de especies $\mathrm{i}$ en la comunidad $\mathrm{B}, \mathrm{N}_{\mathrm{A}}=\Sigma \mathrm{X}_{\mathrm{i}}, \mathrm{N}_{\mathrm{B}}=\Sigma \mathrm{Y}_{\mathrm{i}}$. Un valor de $\mathrm{C}_{\mathrm{H}}=0$ indica que las especies estudiadas consumen diferentes tipos de ítemes alimenticios dentro y entre las estaciones de estudio; $\mathrm{C}_{\mathrm{H}}=1$ indica que las especies estudiadas consumen alimentos similares dentro y entre los períodos de estudio.

\section{RESULTADOS}

La cobertura foliar del estrato herbáceo y arbustivo del sector de estudio no presentó cambios importantes en su abundancia y en el número de especies de plantas durante las dos estaciones contrastadas. La cobertura promedio de la vegetación fue en invierno del $46,5 \%$ y en verano $68,0 \%$ (Tabla 1 ).

Lagidium viscacia fue la especie que consumió el mayor número de ítemes alimenticios: 10 en invierno y siete en verano, de las 12 especies presentes en el sitio de estudio (Tabla 2). Mientras que Abrocoma cinerea consumió cuatro y cinco ítemes durante invierno y verano, respectivamente (Tabla 2). 


\section{TABLA 2}

Itemes alimenticios para el total de campos microscópicos submuestreados (\%) de L. viscacia y A. cinerea (invierno 1996 y verano 1997)

Food items for the total subsampled microscopic fields (\%) of Lagidium viscacia and Abrocoma cinerea (winter 1996 and austral summer 1997)

\begin{tabular}{|c|c|c|c|c|}
\hline \multirow{3}{*}{ Familia y especie } & \multicolumn{4}{|c|}{ Itemes alimenticios } \\
\hline & \multicolumn{2}{|c|}{ L. viscacia } & \multicolumn{2}{|c|}{ A. cinerea } \\
\hline & Invierno & Verano & Invierno & Verano \\
\hline \multicolumn{5}{|l|}{ Compositae } \\
\hline Artemisia copa & 1,1 & - - & - & - \\
\hline Baccharis tola & —— & 0,8 & 73,3 & 33,7 \\
\hline Parastrephia quadrangularis & 9,4 & - - & 0,4 & 2,3 \\
\hline \multicolumn{5}{|l|}{ Ephedraceae } \\
\hline Ephedra rupestris & 0,5 & - & - & 2,9 \\
\hline \multicolumn{5}{|l|}{ Gramineae } \\
\hline Festuca orthophylla & - & 19,9 & - & - \\
\hline Stipa bomanii & 20,9 & 30,1 & - & - - \\
\hline \multicolumn{5}{|l|}{ Papilionaceae } \\
\hline Adesmia horrida & 1,1 & 3,2 & 3,6 & 22,5 \\
\hline Lupinus oreophilus & 0,3 & 0,6 & 11,9 & 24,0 \\
\hline \multicolumn{5}{|l|}{ Scrophulariaceae } \\
\hline Calceolaria stellariifolia & 5,0 & 2,3 & - - & - \\
\hline \multicolumn{5}{|l|}{ Solanaceae } \\
\hline Nicotiana longibracteata & 12,1 & - - & - - & - \\
\hline N. petunioides & 0,4 & - & - & - \\
\hline \multicolumn{5}{|l|}{ Verbanaceae } \\
\hline Junellia seriphioides & 0,3 & 2,0 & - - & - \\
\hline Fibras & 30,9 & 39,8 & 2,4 & 14,3 \\
\hline Material no reconocido & 18,0 & 1,3 & 8,4 & 0,3 \\
\hline
\end{tabular}

En general, L. viscacia mostró una amplitud de nicho trófico mayor que $A$. cinerea durante invierno $\mathrm{H}^{\prime}=0,67$ (Tabla 3 ), donde consumió $10 \mathrm{de}$ las 12 especies presentes en el sitio de estudio (Tabla 2), mientras que en verano, el número de ítemes alimenticios consumidos se redujo a siete especies $\left(\mathrm{H}^{\prime}=0,53\right)$, similar a lo obtenido para $A$. cinerea (Tabla 3 ). Sin embargo, mostró una alta sobreposición trófica entre ambos períodos $\left(\mathrm{C}_{\mathrm{H}}=\right.$ $0,56)$.

Abrocoma cinerea durante invierno tuvo una amplitud del nicho trófico escasa, en comparación a la que presentó en verano (Tabla 3 ). La baja amplitud de nicho trófico exhibida por A. cinerea es consistente con la alta sobreposición trófica que tuvo entre ambos períodos estacionales $\left(\mathrm{C}_{\mathrm{H}}=\right.$ $0,85)$.

De la gran variedad de ítemes alimenticios consumidos por L. viscacia en invierno se destacaron, en términos de selección las herbáceas $N$. longibracteata, Calceolaria stellarifolia, $P$. quadrangularis y $S$. bomanii, mientras que en verano fueron $F$. orthophylla y $S$. bomanii, y en

baja magnitud, A. horrida y C. stellarifolia (Fig. 1).
Abrocoma cinerea durante invierno y verano presentó una alta selección por $L$. oreophilus (Fig. 2). A pesar que el arbusto B. tola fue altamente consumido en ambas estaciones (Tabla 2), presentó baja magnitud de selección (Fig. 2). Hubo un bajo consumo de $A$. horrida, en invierno $(3,6 \%)$, además, mostró rechazo, mientras que en

TABLA 3

Amplitud del nicho trófico (índice de diversidad ecológica de Shannon-Weaver) y símilitud trófica (índice de Horn) de Lagidium viscacia (Lv) y Abrocoma cinerea (Ac) durante invierno 1996 y verano 1997

Trophic niche breadth (Shannon-Weaver's ecological diversity index) and trophic overlap (Horn's index) of Lagidium viscacia and Abrocoma cinerea during winter 1996 and austral summer 1997

\begin{tabular}{|c|c|c|c|c|}
\hline \multirow[b]{2}{*}{ Índice } & \multicolumn{2}{|c|}{ Invierno } & \multicolumn{2}{|c|}{ Verano } \\
\hline & $\mathrm{Lv}$ & Ac & $\mathrm{Lv}$ & $\mathrm{Ac}$ \\
\hline $\begin{array}{l}\text { Shannon-Weaver }\left(\mathrm{H}^{*}\right) \\
\text { Horn }(\mathrm{C})\end{array}$ & $\begin{array}{r}0,67 \\
0\end{array}$ & 0,25 & $\begin{array}{r}0,53 \\
0\end{array}$ & 0,56 \\
\hline
\end{tabular}




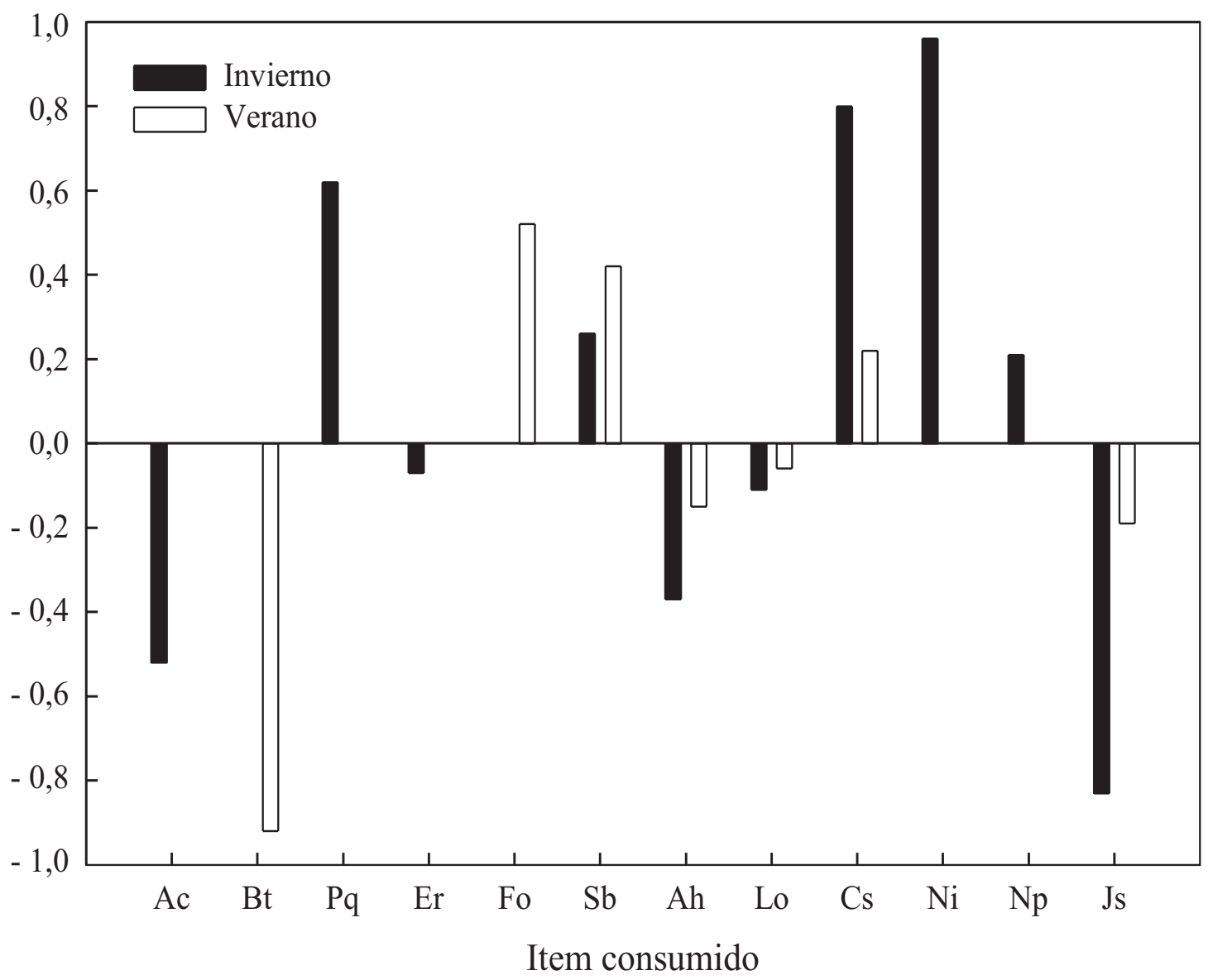

Fig. 1: Selección de ítemes alimenticios (índice de electividad de Ivlev) de Lagidium viscacia durante invierno (1996) y verano (1997) en el Norte Grande de Chile. Nomenclatura de ítemes de plantas consumidas: Artemisia copa $=$ Ac, Baccharis tola $=\mathrm{Bt}$, Parastrephia quadrangularis $=\mathrm{Pq}$, Ephedra rupestris $=\mathrm{Er}$, Festuca orthophylla $=$ Fo, Stipa bomanii $=\mathrm{Sb}$, Adesmia horrida $=\mathrm{Ah}$, Lupinus oreophilus $=\mathrm{Lo}$, Calceolaria stellariifolia $=\mathrm{Cs}$, Nicotiana longibracteata $=\mathrm{Ni}, N$. petunioides $=\mathrm{Np}$, Junellia seriphioides $=\mathrm{Js}$.

Food selection (Ivlev's electivity index) of Lagidium viscacia during winter (1996) and austral summer (1997) in Chile's Norte Grande. Names of plants eaten: Artemisia copa $=\mathrm{Ac}$, Baccharis tola $=\mathrm{Bt}$, Parastrephia quadrangularis $=\mathrm{Pq}$, Ephedra rupestris $=\mathrm{Er}$, Festuca orthophylla $=\mathrm{Fo}$, Stipa bomanii $=\mathrm{Sb}$, Adesmia horrida $=\mathrm{Ah}$, Lupinus oreophilus $=\mathrm{Lo}$, Calceolaria stellariifolia $=\mathrm{Cs}$, Nicotiana longibracteata $=\mathrm{Ni}, N$. petunioides $=\mathrm{Np}$, Junellia seriphioides $=\mathrm{Js}$.

verano fue altamente consumida $(24 \%)$ e, incluso fue seleccionada (Fig. 2). P. quadrangularis, que fue consumida en bajo porcentaje durante invierno $(0,4 \%)$ y verano $(2,3 \%)$, presentó también un alto rechazo en ambas estaciones (invierno: $\mathrm{E}_{\mathrm{i}}=$ 0,82; verano: $\left.\mathrm{E}_{\mathrm{i}}=-0,40\right)$. El arbusto E. rupestris fue consumido sólo en verano $(2,9 \%)$ y mostró selección (Fig. 2).

Pese a que L. viscacia y A. cinerea son roedores que coexisten en sintopía en el sector altoandino en estudio, mostraron durante las dos estaciones contrastantes una baja sobreposición trófica en sus dietas: $\mathrm{C}_{\mathrm{H}}=0,18$ en invierno y $\mathrm{C}_{\mathrm{H}}=0,06$ en verano (Tabla 3 ).

\section{DISCUSIÓN}

Los hábitos alimenticios de roedores generalmente son evaluados por análisis de materiales fecales y/o de los contenidos de estómagos. Ambos análisis ofrecen ciertas desventajas. El principal inconveniente del análisis fecal es que los alimentos, al pasar por el tracto digestivo completo, pueden presentar grados de digestibilidad diferenciales, de modo que los resultados que se obtengan pueden ser sesgados (Jeuniaux 1961, Hansson 1970, Dickman \& Huang 1988, Bergman \& Krebs 1993). La desventaja mayor del análisis de los contenidos estomacales es que se requiere a menudo el sacrificio de un número importante 


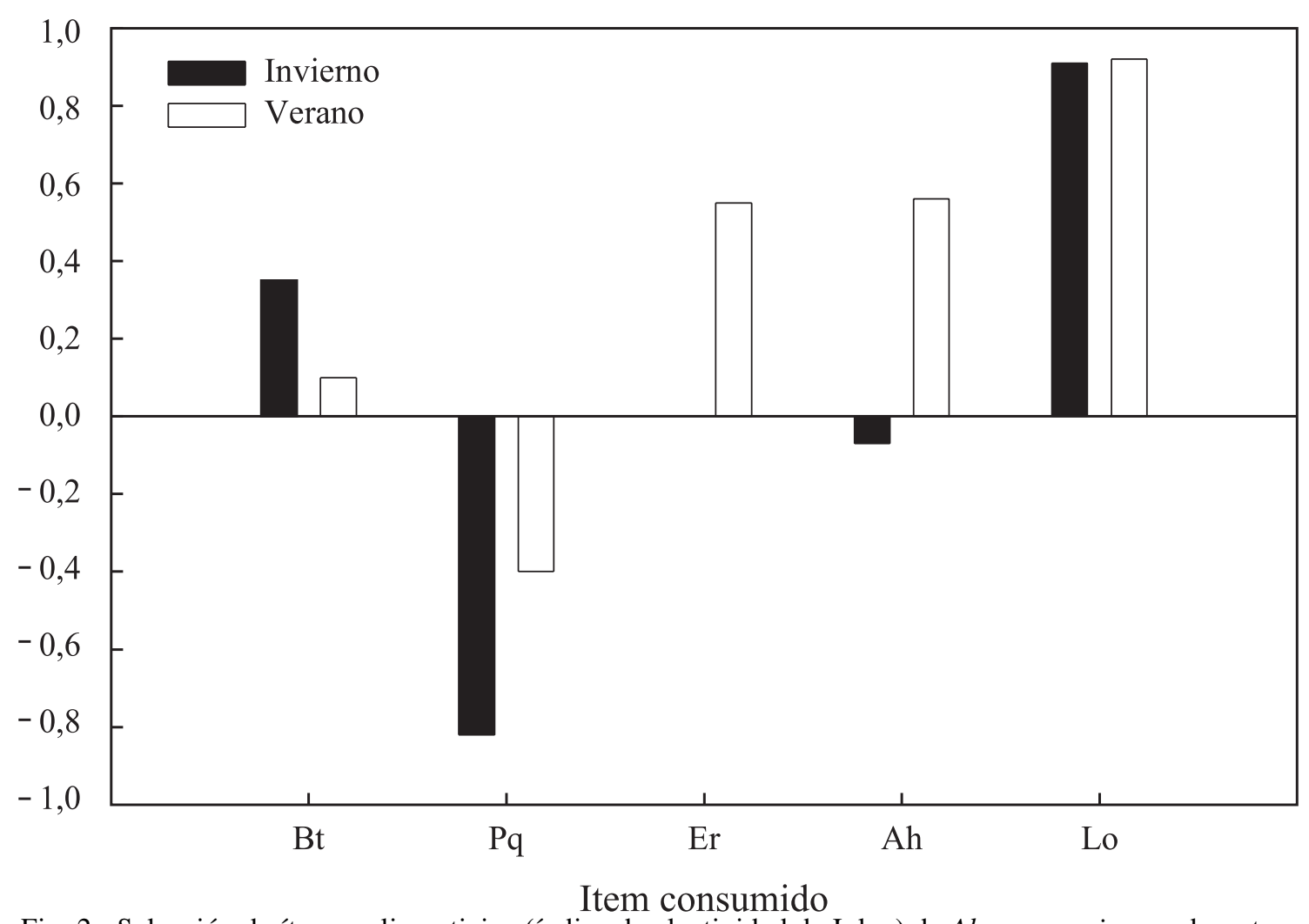

Fig. 2: Selección de ítemes alimenticios (índice de electividad de Ivlev) de Abrocoma cinerea durante invierno (1996) y verano (1997) en el Norte Grande de Chile. Nomenclatura de ítemes de plantas consumidas: Baccharis tola $=\mathrm{Bt}$, Parastrephia quadrangularis $=\mathrm{Pq}$, Ephedra rupestris $=\mathrm{Er}$, Adesmia horrida $=$ Ah y Lupinus oreophilus $=$ Lo.

Food selection (Ivlev's electivity index) of Abrocoma cinerea during winter (1996) and austral summer (1997) in Chile's Norte Grande. Names of eaten plants: Baccharis tola $=\mathrm{Bt}$, Parastrephia quadrangularis $=\mathrm{Pq}$, Ephedra rupestris $=\mathrm{Er}$, Adesmia horrida $=$ Ah y Lupinus oreophilus $=$ Lo.

de animales (véanse Reichman 1975, Bar et al. 1984, Kerley 1992, Tobin et al. 1994, Mushtag et al. 1995), situación que resulta poco factible para estudios de especies con pequeñas poblaciones y, más aún, cuando están bajo cierta amenaza de extinción o protección legal (Kronfeld \& Dayan 1998). Recientemente, se ha propuesto un nuevo método para determinar la dieta de roedores, consistente en el bombeo del estómago; el cual no implica el sacrificio de los animales, de modo que es posible colectar más de una muestra por individuo y, también, es posible estudiar una población de roedores por un largo período de tiempo sin dañarlos (Kronfeld \& Dayan 1998). Sin embargo, para el caso de estudios de campo, esta técnica es poco adecuada, especialmente cuando se trata de poblaciones reducidas de una especie y cuyos individuos son difíciles de capturar, tal como ocurre con Lagidium viscacia y Abrocoma cinerea, especies consideradas en el presente trabajo.

Los roedores $L$. viscacia y A. cinerea son especies que coexisten en sintopía en los ambientes altoandinos del Norte Grande de Chile, caracterizados por presentar escasa vegetación, conformada por plantas arbustivas y herbáceas, distribuidas en forma heterogénea, debido principalmente a la gran cantidad de afloramientos rocosos (Tabla 1). La escasa vegetación presente en el sector de estudio probablemente se deba a las condiciones climáticas extremas de la puna altoandina.

Estudios ecológicos y fisiológicos de estas dos especies son escasos. Se ha descrito que L. viscacia no bebería agua libre, necesidad que sería cubierta a través del agua contenida en las plantas; para A. cinerea sólo se menciona que es un herbívoro (Pearson 1948, Mann 1978). Al respecto, hemos encontrado que los ítems de alimentos mayormente consumidos por L. viscacia fueron las gramíneas Stipa bomanii y Festuca orthophilla (Tabla 2), plantas que presentaron contenidos de agua de $72,4 \%$.

En general, estos roedores presentaron consumos relativamente importante de fibras y tejidos conductores (xilema y floema), particularmente L. viscacia (invierno $=30,9 \%$, verano $=39,8 \%$ ), 
en cambio $A$. cinerea mostró menores consumos $($ invierno $=2,4 \%$, verano $=14,3 \%)($ Tabla 2$)$. Estos porcentajes de fibras y tejidos conductores presentes en la dieta de estas dos especies de roedores podrían deberse a: (1) La utilización del agua contenida en la corteza de las plantas, situación que ha sido observada con frecuencia en roedores que dañan la corteza de Pinus radiata (Rojas et al. 1977, Murúa \& Rodríguez 1985a, 1985b); (2) una sobreestimación de materiales no digeribles, como ocurre con fibras y vasos conductores (xilema) en comparación a las partes blandas, como hojas que son más digeribles (véase Randolph et al. 1991); (3) la menor disponibilidad de recursos alimenticios (y/o nutritivos) durante el verano (años secos), especialmente de partes blandas (hojas), de modo que los animales tendrían que recurrir a un consumo mayor de fibras y vasos conductores.

De las 12 especies de plantas que conformaron la vegetación del sector de estudio, L. viscacia consumió 10 especies en invierno $(83,3 \%)$ y siete especies en verano $(58,3 \%)$, de modo que durante estos dos períodos estacionales utilizó la mayor parte de las especies de plantas presentes en el sitio de estudio (Tabla 1 y 2 ). En contraste, $A$. cinerea sólo consumió cinco especies, equivalentes al $41,7 \%$ (Tabla 1 y 2 ). Con respecto a la disponibilidad de los recursos alimenticios, las herbáceas S. bomanii, F. orthophylla y el arbusto Baccharis tola fueron las especies que presentaron la mayor cobertura vegetacional (Tabla 1). En general, estas especies de plantas fueron las que mostraron los mayores signos de ramoneo y, además, correspondieron a los ítemes alimenticios más consumidos por L. viscacia y A. cinerea (Tabla 2 y 3 ). Sin embargo, debe considerarse que dentro del ensamble taxonómico de roedores presentes en el sitio de estudio, también se encontraron a los múridos Phyllotis rupestris, Abrothrix andinus y Eligmodontia puerulus, micromamíferos que potencialmente podrían consumir los mismos ítemes de plantas (Rau et al. 1998, Spotorno et al. 1998).

Tomando en cuenta los análisis fecales se encontró que L. viscacia fue la especie que presentó la mayor amplitud de nicho trófico, siendo $S$. bomanii la especie que mostró el más alto consumo (20,9 a 30,1\%) (Tabla 2). No obstante, si se consideran las magnitudes del índice de electividad de Ivlev, se observó que en invierno los mayores valores se obtuvieron para Nicotiana longibracteata, Calceolaria stellarifolia y Parastrephia quadrangularis, mientras que en verano fueron principalmente las gramíneas $F$. orthophylla y S. bomanii (Fig. 1), plantas que en el área de estudio presentaron una menor oferta ambiental que el arbusto $B$. tola (Tabla 1). Estos resultados sugieren que L. viscacia poseería selectividad por dichos tipos de alimento (Fig. 1), a pesar que consumió durante las dos estaciones prácticamente la totalidad de las especies de plantas presentes en el sitio de estudio. Planteamiento que contrasta con el amplio nicho trófico que esta especie mostró en invierno y verano $(0,67$ y 0,53 , respectivamente) (Tabla 3). En general, L. viscacia presentó menor sobreposición trófica $\left(\mathrm{C}_{\mathrm{H}}=0,56\right)$ entre invierno y verano que $A$. cinerea $\left(\mathrm{C}_{\mathrm{H}}=0,85\right)$ (Tabla $3)$, lo que indicaría que es un roedor herbívoro más generalista que $A$. cinerea (Tabla 2, Fig. 1 y 2).

Abrocoma cinerea consumió principalmente $B$. tola (Tabla 2), planta arbustiva que constituyó el mayor porcentaje de la cobertura vegetación (Tabla 1). Si uno se basara exclusivamente en dicho porcentaje de consumo como la única fuente de información, se podría inferir que dicho ítem alimenticio sería seleccionado por este roedor. Sin embargo, utilizando el índice de selectividad de Ivlev se encontró que $A$. cinerea presentó mayor selección por Lupinus oreophilus (Fig. 2), planta herbácea que se encontró en baja proporción dentro de la cobertura de la vegetación ( $£$ $0,8 \%$ ) (Tabla 1 ).

En general, $A$. cinerea exhibió una baja amplitud de nicho trófico, especialmente en invierno $\left(\mathrm{H}^{\prime}=\right.$ $0,25)$, cuyo valor fue menos de la mitad que el calculado para verano $\left(\mathrm{H}^{\prime}=0,56\right)$ (Tabla 3$)$. La mayor amplitud de nicho trófico de $A$. cinerea en verano fue coincidente con su período reproductivo, inferencia que está basada en el hecho de que sólo durante el verano se obtuvieron capturas de individuos juveniles (A. Cortés resultados no publicados). Por otra parte, se observó que durante dicho período hubo un mayor consumo de A. horrida y $L$. oreophilus, especies de la familia Papilionaceae (Tabla 1), las que normalmente contienen mayores contenidos de proteínas que otras plantas (Fernandes \& Pereyra 1995). Esto podría favorecer los mayores requerimientos de nutrientes, especialmente en las hembras durante el período de gestación y mantención de las crías.

Abrocoma cinerea presentó un alta sobreposición dietaria $\left(\mathrm{C}_{\mathrm{H}}=0,85\right)$ entre invierno y verano (Tabla 2). Esta similitud trófica era predecible, dado que en el ambiente altoandino estudiado existen escasos recursos alimentarios (cobertura $<50 \%$ ) y un número reducido de especies de plantas potenciales de utilizar como alimento por micromamíferos herbívoros silvestres. A pesar que los recursos alimenticios presentes en el sitio de estudio fueron escasos (Tabla 1), fluctuantes y poco predecibles, no obstante, estas dos especies de roedores mostraron una escasa sobreposición trófica (invierno: $\mathrm{C}_{\mathrm{H}}=0,18$; verano: $\mathrm{C}_{\mathrm{H}}=0,06$; 
Tabla 3); lo que estaría indicando que estas especies sintópicas presentarían una alta segregación trófica. En consecuencia, la presumible competencia explotativa por alimento sería mínima. Por otro lado, ambas especies difieren en sus ritmos de actividad, siendo L. viscacia de hábito diurnocrepuscular, mientras que $A$. cinerea es preferentemente nocturna (Mann 1978, Galende \& Grigera 1998). Además, hemos observado que estas especies ocupan diferentes sectores de roqueríos como refugios, encontrándose $L$. viscacia en roqueríos más altos, mientras que $A$. cinerea ocupa preferentemente sectores de roqueríos más bajos. Sin embargo, parecería que ambas especies se alimentan en las mismas áreas.

En conclusión, en el presente estudio se encontró que L. viscacia y A. cinerea son roedores que coexisten en sintopía en los ambientes áridos altoandinos del norte de Chile, sus hábitos alimentarios son herbívoros, con una baja tendencia a presentar una selección trófica definida, especialmente en el caso de L. viscacia, especie que tendió a ser más generalista. No obstante, ambas especies presentaron nichos tróficos diferentes, y, además, se segregarían temporal (ritmos de actividad) y micro-espacialmente en los sectores de roqueríos en que habitan, de modo que la condición estricta de sintopía para estas especies en el área de estudio no implicaría necesariamente una presunta competencia explotativa por estas tres dimensiones de nicho (cuantitativa y cualitativamente), por lo menos no durante las dos estaciones estudiadas.

\section{AGRADECIMIENTOS}

Agradecemos a la profesora Gina Arancio, conservadora del Herbario de la Universidad de La Serena, por su valiosa colaboración en la identificación de las especies de plantas del sitio de estudio, y al Dr. J. Gutiérrez por la lectura crítica del manuscrito. Este trabajo fue financiado por el proyecto FONDECYT 5960017, del Programa Sectorial Biomas y Climas del Norte de Chile, Comisión Nacional de Investigación Científica y Tecnológica de Chile (CONICYT) y el proyecto FONDECYT 1981122.

\section{LITERATURA CITADA}

ALCOZE TM \& EG ZIMMERMAN (1973) Food habits and dietary overlap of two heteromyid rodents from the mesquite plains of Texas. Journal of Mammalogy 54: $900-908$
ALIPAYOU D, JL HOLECHEK, R VALDEZ, A TEMBO, L. SAIWANA, M. RUSCO \& M CARDENAS (1993) Range condition influences on Chihuahuan Desert cattle and jackrabbit diets. Journal of Wildlife Management 46: 296-301.

BAR Y, Z. ABRAMSKY \& Y GUTERMAN (1984) Diet of gerbilline rodents in the Israeli desert. Journal of Arid Environment 7: 371-376.

BEG MA, AA KHAN, UI MUSHTAG \& M HASSAN (1994) Food habits of Millardia meltada (Rodentia, Muridae) in the croplands of central Punjab (Pakistan). Mammalia 58: 242-253

BELOVSKY GE (1978) Diet optimization in a generalist herbivore: the moose. Theoretical Population Biology 14: $105-134$

BELOVSKY GE (1984) Herbivore optimal foraging: comparative test of three models. The American Naturalist 124: 97-115.

BERGMAN CM \& CJ KREBS (1993) Diet overlap of collared lemmings and tundra voles at Pearce Point, Northwest Territories. Canadian Journal of Zoology 71: 1703-1709.

BRIGHT PW \& PA MORRIS (1994). Animal translocation for conservation: perfomance of dormice in relation to release methods, origin and season. Journal of Applied Ecology 31: 699-708.

CAMPOS H (1996) Mamíferos terrestres de Chile. Corporación Nacional Forestal \& María Cúneo Ediciones, Valdivia, Chile. 248 pp.

COX GW (1990) Laboratory manual of general ecology. Sixth edition. Wm. C. Brown Publishers, Dubuque, Iowa. $251 \mathrm{pp}$

COLE FR, LL LOOP, AC MEDEIROS, JA RAIKES \& CS WOOD (1995) Conservation implications of introduced game birds in high-elevation Hawaiian shrubland. Conservation Biology 9: 306-313.

CRAWLEY MJ (1983) Herbivory: the dynamics of animal-plant interactions. Studies in Ecology. University of California Press, Berkeley, California 10: 1-437.

DALKE PD, WK CLARK, JR \& LJ KORSCHGEN (1942) Food habit trends of the wild turkey in Missouri as determined by dropping analysis. Journal of Wildlife Management 6: 237-243.

DAVISON VE \& KE GRAETZ (1957) Managing habitat for white-tailed deer and wild turkeys. Transactions of the North American Wildlife Natural Resources Conference 22: 412-424.

DICKMAN CR \& C HUANG (1988) The reliability of fecal analysis as a method for determining the diet of insectivorous mammals. Journal of Mammalogy 69: 108-113.

DIZEO DE STRITTMATTER CG (1984) Métodos de clarificación en materiales vegetales. Parodiana 3: 169174.

DRICKAMER L (1970) Seed preferences in wild caught Peromyscus maniculatus bairdii and Peromyscus leucopus noveboracensis. Journal of Mammalogy 51: 191-194.

DRICKAMER L (1971) Experience and selection behaviour in the food habits of Peromyscus: use of olfaction. Behaviour 61: 269-287. 
DUSI JL (1949) Methods for determination of food habits by plant microtechnics and histology and their application to cottontail rabbit food habits. Journal of Wildlife Management 13: 295-298.

FERNANDES MS \& RO PEREYRA (1995) Mineral nitrogen in plant physiology and nutrition. Critical Reviews in Plant Science 14: 111-148.

FITZGERALD AE \& DC WADDINGTON (1979) Comparison of two methods of fecal analysis of herbivore diet. Journal of Wildlife Management 43: 468-473.

GLADE A ed (1993). Libro rojo de vertebrados terrestres de Chile. Corporación Nacional Forestal, Ministerio de Agricultura, Santiago, Chile. 65 pp.

GALENDE GI \& D GRIGERA (1998) Relaciones alimentarias de Lagidium viscacia (Rodentia, Chinchillidae) con herbívoros introducidos en el Parque Nacional Nahuel Huapi, Argentina. Inheringia Série Zoología 84: 3-10.

GROSJEAN M, B MESSERLI \& H SCHREIER (1991) Seenhoschstände, Bodenbildug und Vergletscherung im Altiplano Nordchiles: Ein interdisziplinärer Beitrag zur Klima geschichte der Atacama. Erste Resultate. In "Südamerika Geomorphologie und Paläoökologie im jüngeren Quartär”, Bamberger Geographische Schriften 11: 99-108.

HANSSON L (1970) Methods of morphological diet microanalysis in rodents. Oikos 21: 255-266.

HARBORNE JB (1982) Introduction to ecological biochemistry. Second edition. Academic Press, London, England. 278 pp.

HARTMAN G (1994) Long-term population development of a reintroduced beaver (Castor fiber). Conservation Biology 8: 713-717.

HOLECHEK JL, M VAVRA \& RD PIEPER (1982a) Botanical composition determination of range herbivore diets: a review. Journal of Wildlife Management 35: 309-315.

HOLECHEK JL, BD GROSS, SM DABO \& T STEPHENSON (1982b) Effects of sample preparation, growth stage, and observer on microhistological analysis of herbivore diets. Journal Wildlife of Management 46: 502-505.

HORN HS (1966) Measurement of overlap in comparative ecological studies. American Naturalist 100: 419424.

HURTS GA (1992) The wild turkey: biology and management. En: Dickson JG (ed) Food and feeding: 63-83. Stackpole Books, Harrisburg, Pennsylvania.

JIMENEZ R, JA HODAR \& I CAMACHO (1994) Diet of the woodpigeon (Columba palumbus) in the south of Spain during late summer. Folia Zoolica 43: 163-170.

JEUNIAUX C (1961) Chitinase: an addition to the list of hydrolases in the digestive tract of vertebrates. Nature 192: 135-136.

KERLEY GIH (1992) Trophic status of small mammals in the semi-arid Karoo. South African Journal of Zoology 226: 563-572.

KORSCHGEN LJ (1969) Procedures for food-habits analyses. En: Giles RH Jr (ed) Wildlife management techniques: 233-250. The Wildlife Society, Washington, District of Columbia.

KREBS CJ (1989) Ecological methodology. Harper Collins Publisher, New York, New York. 654 pp.
KRONFELD N \& T DAYAN (1998) A new method of determining diets of rodents. Journal of Mammalogy 79: 1198-1202.

LEWIS SW (1994) Fecal and rumen analyses in relation to temporal variation in black-tailed deer diets. Journal of Wildlife Management 58: 53-58.

LUNDIE-JENKINS G, LK CORBETT \& CM PHILLIPS (1993) Ecology of the rufous hare-wallaby, Lagorchester hirsutus Gould (Marsupialia: Macropodidae), in the Tanami Desert, Northern Territory: III. Interactions with introduced mammal species. Wildlife Research 20: 495-511.

MANN G (1978) Los pequeños mamíferos de Chile: marsupiales, quirópteros y edentados. Gayana Zoología (Chile) 40: 1-342.

MARQUET PA, F BOZINOVIC, GA BRADSHAW, C CORNELIUS, H GONZÁLEZ, JR GUTIÉRREZ, ER HAJEK, JA LAGOS, F LÓPEZ-CORTÉS, H SAMANIEGO, VG STANDEN, JC TORRES-MURA \& FM JAKSIC (1998) Los ecosistemas del desierto de Atacama y áreas andinas adyacente en el norte de Chile. Revista Chilena de Historia Natural 71: 593-617.

MESERVE PL (1981) Trophic relationship among small mammals in a Chilean semiarid thorn scrub community. Journal of Mammalogy 62: 304-314.

MORALES A (1985) Análisis cuantitativo de las dietas del ganado vacuno y venado cola blanca en la Michalía, Durango. Tesis de Licenciatura, Facultad de Ciencias, Universidad Nacional Autónoma de México, México, Distrito Federal. 107 pp.

MUELLER-DOMBOIS D \& H ELLEMBERG (1974) Aims and methods of vegetation ecology. John Wiley \& Sons, New York, New York. 547 pp.

MURÚA R \& J RODRÍGUEZ (1985a) Evaluación y alternativas de control del daño ocasionado por roedores en plantaciones de pino insigne (Pinus radiata, D. Don), en la VII y VIII Región. Informe Final Proyecto CONAF y Empresas Forestales. $91 \mathrm{pp}$.

MURÚA R \& J RODRÍGUEZ (1985b) Características del daño por roedores en plantaciones de pino (Pinus radiata, D. Don). En: Olivares B \& F Morales (eds) Pinus radiata investigación en Chile: 247-258. Universidad Austral de Chile, Valdivia, Chile.

MUSHTAG UL, M HASSAN, MA BEG, AA KHAN, SR HUSSAIN \& SM KHAN (1995) Food habits of the house rat (Rattus rattus) in rural central Punjab (Pakistan). Pakistan Journal of Zoology 27: 115-118.

OWEN-SMITH N \& P NOVELLIE (1982) What should a clever ungulate eat? American Naturalist 119: 151178.

PEARSON OP (1948) Life history of mountain viscacha in Perú. Journal of Mammalogy 29: 345-374.

PERKIN JL (1982) Shannon-Weaver or Shannon-Weiner? Journal of Water and Pollution Contribution 54: 10491050 .

PETRIDES G (1975) Principal foods versus preferred foods and their relation to stocking rate and range condition. Biological Conservation 7: 161-169.

RAU RJ, C ZULETA, A GANTZ, F SAIZ, A CORTES, L YATES, AE SPOTORNO \& E COUVE (1998) Biodiversidad de artrópodos y vertebrados terrestres del Norte Grande de Chile. Revista Chilena de Historia Natural 71: 527-554. 
RANDOLPH JC, GN CAMER0N \& JA WRAZEN (1991)

Dietary choice of a generalist grassland hervivore, Sigmodon hispidus. Journal of Mammalogy 72: 300313.

REDFORD KH \& JF EISENBERG (1992) Mammals of the Neotropics: the southern cone. The University of Chicago Press, Chicago, Illinois. 430 pp.

REICHMAN OJ (1975) Desert rodent diets. Journal of Mammalogy 56: 731-735.

RENSSEN TA \& RL VOGEL (1993) Recent developments of the raven Corvus corex population in The Netherlands. Limosa 66: 107-116.

ROJAS M, O RIVERA, G MONTENEGRO \& C BARROS (1977) Algunas observaciones en la reproducción de la hembra silvestre de Octodon degus (Molina) y su posible relación con la fenología de la vegetación. Medio Ambiente (Chile) 3: 78-82.

SEVERSON K E (1981) Food habits and nutritional relationships of Mule deer in southwestern United States. En: Folliot PF \& S Gallina (eds) Deer biology, habitat requirement, and management in western North America: 4: 149-164. Instituto de Ecología AC, México Distrito Federal.

SPARK DR \& JC MALECHEK (1968) Estimating percentage dry weight in diets using microscopic technique. Journal of Wildlife Management 21: 264265.
SPOTORNO AE, C ZULETA, A GANTZ, F SAIZ, J RAU, M ROSENMANN, A CORTES, GRUIZ, L YATES, E COUVE \& JC MARIN (1998) Sistemática y adaptación de mamíferos, aves e insectos fitófagos de la Región de Antofagasta, Chile. Revista Chilena de Historia Natural 71: 501-526.

STEWART DRM (1967) Análisis of plant epidermis in faeces: a technique for studying the foraging preferences of grazing herbivores. Journal of Applied Ecology 4: 83-111.

TOBIN ME, AE KOEHLER \& RT SUGIHARA (1994) Seasonal patterns of fecundity and diet of roof rats in Hawaiian macademia orchards. Wildlife Research 21: 519-526.

WESTOBY M (1978) What are the biological bases of varied diets? American Naturalist 112: 627-631.

WILLIAMS O (1962) A technique for studying microtine food habits. Journal of Mammalogy 43: 365-368.

ZIMMERMAN EG (1965) A comparison of habitat and food the two species of Microtus. Journal of Mammalogy 46: 605-612. 